\title{
Maternal high-fat hypercaloric diet during pregnancy results in persistent metabolic and respiratory abnormalities in offspring
}

\author{
Pamela S. Griffiths ${ }^{1}$, Cheryl Walton ${ }^{1}$, Lennie Samsell ${ }^{1}$, Miriam K. Perez ${ }^{2}$ and Giovanni Piedimonte ${ }^{3}$
}

BACKGROUND: We have shown in a previous populationbased study significant correlation between childhood asthma and early abnormalities of lipid and glucose metabolism. This study's specific aim was to determine whether maternal nutrition in pregnancy affects postnatal metabolic and respiratory outcomes in the offspring.

METHODS: On gestation day 1, dams were switched from standard chow to either high-fat hypercaloric diet or control diet. Terminal experiments were performed on newborn and weanling offspring of dams fed the study diet during gestation and lactation, and on adult offspring maintained on the same diet as their mother.

RESULTS: Pups born from high-fat hypercaloric diet (HFD) dams developed metabolic abnormalities persistent throughout development. Cytokine expression analysis of lung tissues from newborns born to HFD dams revealed a strong proinflammatory pattern. Gene expression of neurotrophic factors and receptors was upregulated in lungs of weanlings born to HFD dams, and this was associated to higher respiratory system resistance and lower compliance at baseline, as well as hyperreactivity to aerosolized methacholine. Furthermore, HFD dams delivered pups prone to develop more severe disease after respiratory syncytial virus (RSV) infection.

CONCLUSION: Maternal nutrition in pregnancy is a critical determinant of airway inflammation and hyperreactivity in offspring and also increases risk for bronchiolitis independent from prepregnancy nutrition.

C urrent epidemiologic data indicate that $17 \%$ of children in the United States are obese, while $9.6 \%$ have been diagnosed with asthma (1). The parallel rise in the prevalence of these two conditions in industrialized countries over the last decades has led several investigators to propose pathophysiologic links, but the causality of this relationship remains controversial (2). We have previously described the relationship between asthma and body mass in a sample of 18,000 schoolage children across the entire range of weight percentile categories, showing that children with asthma tend to have higher serum triglyceride levels and higher rates of insulin resistance regardless of the children's body mass. Thus, early abnormalities of lipid and glucose metabolism may be associated with the development of asthma and confound its epidemiologic link to obesity (3).

Both obesity and asthma likely have their beginnings in utero and early childhood, and nutritional factorsespecially prenatal and early infant diet-may play a role in their pathogenesis. The plausibility of a pathophysiologic link between maternal metabolism and early airway dysfunction is supported by data in preterm infants exposed to antenatal inflammation in the form of chorioamnionitis, which increases the odds of developing chronic lung disease (4). While to a lesser extent, chronic low-grade inflammation and immune system activation play a critical role in the pathogenesis of obesity and its metabolic complications (5), and similar inflammatory patterns have been found in response to high-fat diet (6). Thus, fetal exposure to imbalanced maternal diet could result in a predisposition to pathological airway responses to environmental challenges even in the absence of prepregnancy maternal obesity.

In this study, we test the hypothesis that maternal high-fat hypercaloric diet (HFD) interferes with fetal lung development leading to persistent airway inflammation and hyperreactivity in the offspring through overexpression of proinflammatory cytokines and growth factors. Since diet manipulation in pregnant women is neither ethically nor practically possible, we developed an animal model using a rat strain without genetic predisposition to obesity and considered ideal for immunonutrition studies (7). In this model, we fed dams with HFD or control diet (CD) during pregnancy and lactation and compared the consequences in their offspring in terms of metabolic profiles, inflammatory status, neurotrophic pathways, and lung function in early vs. adult life. In addition, we compared the effects of early-life exposure of offspring from HFD and $\mathrm{CD}$ dams to the most common respiratory pathogen in infancy-respiratory syncytial virus (RSV) - to evaluate the interactions between maternal and environmental factors on postnatal lung function. 


\section{RESULTS}

\section{Body Composition and Biochemistry}

Average birth weight was identical in newborns born to HFD dams compared to age-matched controls born to CD dams, although their total cholesterol, triglycerides, and glucose serum concentrations were significantly lower (Table 1). In contrast, weanlings born to HFD dams became significantly lighter than age-matched controls, but they also tended to have increased body fat percentage. This trend became highly statistically significant in the adult rats born to HFD dams, which also had higher serum triglycerides and showed a trend to become heavier than age-matched controls, whereas total and high-density lipoprotein cholesterol and glucose were not significantly different.

\section{Cytokines and Chemokines}

Multiple proinflammatory cytokines and chemokines were significantly increased during the experiment in offspring born to HFD mothers compared to age-matched controls born to $\mathrm{CD}$ mothers, with important differences depending on age and sampling site. In newborns delivered from HFD dams, lung inflammation was driven by interleukin (IL)- $1 \alpha$, IL-1 $\beta$, IL-2, IL-5, IL-9, eotaxin, tumor necrosis factor $\alpha$ (TNF- $\alpha$ ), and monocyte chemoattractant protein-1 (MCP-1) (Table 2). In weanlings born to HFD dams, the most significant changes were an average 50-fold increase in serum levels of IL-5 and a 5-fold increase in MCP-1 (Table 3); the same cytokines were significantly increased in the lung tissues, and the latter also in bronchoalveolar lavage (BAL) obtained from the same HFD weanlings (Table 4). In adult rats born to HFD dams, only leptin was significantly increased in serum compared with age-matched controls born to CD dams, and this increase was measured also in the BAL obtained from the same rats together with a smaller increase in IL-17.

\section{Neurotrophins}

Prenatal nutrition had profound effects on the expression of key growth factors that modulate structure and function of the sensory and motor innervation in the offspring respiratory tract. Both nerve growth factor (NGF) and brain-derived neurotrophic factor (BDNF) genes were upregulated in the lung tissues of pups born to HFD dams, but the transcripts encoding BDNF increased fourfolds (Figure 1; $n=7$ per group; $P<$ 0.001), whereas the increase of NGF transcripts was smaller and did not reach statistical significance. On the other hand, the largest change was measured for the tropomyosin-related kinase receptor A (TrKA) high-affinity receptor of NGF that increased 25 -folds $(P<0.01)$, while a smaller nonsignificant increase was measured for the tropomyosin-related kinase receptor $B(\operatorname{TrKB})$ high-affinity receptor of BDNF. Also significantly increased was the nonspecific pan-neurotrophin receptor $\mathrm{p} 75^{\mathrm{NTR}}(P<0.001)$.

\section{Pulmonary Function}

In previous studies performed in the same animal model, we have shown that the neurotrophic pathways described above control airway smooth muscle tone and contraction in response to electrical and cholinergic stimulation. Consistently, weanlings born to HFD dams had significantly higher respiratory system resistance $\left(\mathrm{R}_{\mathrm{rs}} ; P<0.01\right)$ and lower respiratory system compliance $\left(\mathrm{C}_{\mathrm{rs}} ; P<0.001\right)$ at baseline compared with

Table 1. Body composition and biochemistry in offspring

\begin{tabular}{|c|c|c|c|c|}
\hline \multicolumn{2}{|r|}{ Variable } & \multirow{2}{*}{$\begin{array}{c}\text { Maternal control diet } \\
5.3 \pm 0.06\end{array}$} & \multirow{2}{*}{$\begin{array}{c}\text { Maternal high-fat diet } \\
5.3 \pm 0.06\end{array}$} & \multirow{2}{*}{$\begin{array}{r}P \text { value } \\
0.927\end{array}$} \\
\hline Newborns & Body weight (g); $C D n=13 ; \mathrm{HFD} n=13$ & & & \\
\hline & Body fat (\%) & NA & NA & NA \\
\hline & Total cholesterol $(\mathrm{mg} / \mathrm{dl}) ; \mathrm{CD} n=6 ; \mathrm{HFD} n=8$ & $114.0(106.0,119.0)$ & $100($ all $<100)$ & $<0.001$ \\
\hline & HDL cholesterol (mg/dl) & NA & NA & NA \\
\hline & Glucose (mg/dl); CD $n=6 ; \mathrm{HFD} n=9$ & $112.2 \pm 12.1$ & $72.0 \pm 2.9$ & 0.002 \\
\hline & Triglycerides (mg/dl), CD $n=6 ; \mathrm{HFD} n=9$ & $263.5 \pm 33.2$ & $138.7 \pm 19.7$ & 0.004 \\
\hline \multirow[t]{6}{*}{ Weanlings } & Body weight $(\mathrm{g}) ; \mathrm{CD} n=7 ; \mathrm{HFD} n=6$ & $29.9 \pm 0.26$ & $23.7 \pm 0.42$ & $<0.001$ \\
\hline & Body fat (\%); CD $n=7 ; \mathrm{HFD} n=7$ & $0.42 \pm 0.03$ & $0.50 \pm 0.03$ & 0.052 \\
\hline & Total cholesterol $(\mathrm{mg} / \mathrm{dl}) ; \mathrm{CD} n=4 ; \mathrm{HFD} n=6$ & $139.5(125.8,149.5)$ & $123(121.5,124.3)$ & 0.762 \\
\hline & HDL cholesterol (mg/dl); CD $n=4 ; \mathrm{HFD} n=3$ & $54.0 \pm 13.2$ & NA & NA \\
\hline & Glucose (mg/dl); CD $n=4 ; \mathrm{HFD} n=6$ & $132.5 \pm 19.5$ & $156.8 \pm 9.2$ & 0.510 \\
\hline & Triglycerides (mg/dl); CD $n=4 ; \mathrm{HFD} n=6$ & $227.0(210.0,322.5)$ & $650(650,650)$ & 0.010 \\
\hline \multirow[t]{6}{*}{ Adults } & Body weight $(\mathrm{g}) ; \mathrm{CD} n=7 ; \mathrm{HFD} n=9$ & $298.0(195.0,320.0)$ & $354.0(262.5,382.0)$ & 0.056 \\
\hline & Body fat (\%); CD $n=7 ; \mathrm{HFD} n=9$ & $5.26 \pm 0.77$ & $8.70 \pm 0.47$ & $<0.001$ \\
\hline & Total cholesterol $(\mathrm{mg} / \mathrm{dl}) ; \mathrm{CD} n=7 ; \mathrm{HFD} n=7$ & $100(100,100)$ & $100(100,100)$ & 1.00 \\
\hline & $\mathrm{HDL}$ cholesterol (mg/dl); CD $n=7, \mathrm{HFD} n=6$ & $49.0(43.0,53.0)$ & $41.0(34.5,46.5)$ & 0.073 \\
\hline & Glucose (mg/dl); CD $n=7, \mathrm{HFD} n=7$ & $174.3 \pm 24.5$ & $207.9 \pm 25.2$ & 0.359 \\
\hline & Triglycerides $(\mathrm{mg} / \mathrm{dll}) ; \mathrm{CD} n=7 ; \mathrm{HFD} n=7$ & $106.6 \pm 22.7$ & $190.57 \pm 26.3$ & 0.032 \\
\hline
\end{tabular}


age-matched controls born to CD dams (Figure 2; $n=7$ per group). Following challenge with aerosolized methacholine, significantly higher airway resistance and lower lung compliance were observed in HFD offspring with cumulative doses up to $8.75 \mathrm{mg} / \mathrm{ml}$. In adult offspring, there were no significant differences in baseline resistance or compliance, but $\mathrm{R}_{\mathrm{rs}}$ increased significantly more in offspring from HFD mothers

Table 2. Significant increase of cytokines and chemokines in lung tissues of offspring

\begin{tabular}{|c|c|c|c|c|}
\hline & Analyte & $\begin{array}{l}\text { Control diet } \\
(\mathrm{pg} / \mathrm{ml})\end{array}$ & $\begin{array}{l}\text { High-fat diet } \\
\quad(\mathrm{pg} / \mathrm{ml})\end{array}$ & $P$ value \\
\hline \multirow{8}{*}{$\begin{array}{l}\text { Newborns; } \\
\text { CD } n=7 \\
\text { HFD } n=7\end{array}$} & Eotaxin & $29.5(27.0,31.4)$ & $38.7(33.5,49.3)$ & 0.011 \\
\hline & IL-1 $\alpha$ & $4.0(3.0,4.6)$ & $15.2(10.7,25.2)$ & $<0.001$ \\
\hline & IL-1 $\beta$ & $6.9(6.2,7.5)$ & $14.4(13.1,18.4)$ & $<0.001$ \\
\hline & IL-2 & $139.0 \pm 7.3$ & $239.0 \pm 21.3$ & $<0.001$ \\
\hline & IL-5 & $1.4(1.2,2.0)$ & $2.8(2.1,4.2)$ & 0.002 \\
\hline & IL-9 & $\begin{array}{c}368.1 \\
(316.6,426.0)\end{array}$ & $\begin{array}{c}476.0 \\
(394.2,626.2)\end{array}$ & 0.017 \\
\hline & TNF- $\alpha$ & $42.7 \pm 3.1$ & $120.8 \pm 7.4$ & $<0.001$ \\
\hline & MCP-1 & $22.3 \pm 0.8$ & $26.7 \pm 1.5$ & 0.026 \\
\hline \multirow{4}{*}{$\begin{array}{l}\text { Weanlings; } \\
\text { CD } n=7 ; \\
\text { HFD } n=7\end{array}$} & IL-5 & $0.12 \pm 0.03$ & $0.44 \pm 0.07$ & 0.001 \\
\hline & TNF- $\alpha$ & $1.02 \pm 0.06$ & $1.55 \pm 0.10$ & $<0.001$ \\
\hline & TGF- $\beta 2$ & $2.17 \pm 0.32$ & $4.02 \pm 0.63$ & 0.01 \\
\hline & MCP-1 & $1.74(1.19,2.22)$ & $6.81(5.62,8.47)$ & $<0.001$ \\
\hline \multirow{3}{*}{$\begin{array}{l}\text { Adults; } \\
\text { CD } n=7 ; \\
\text { HFD } n=9\end{array}$} & IL-18 & $66.27 \pm 3.94$ & $83.0 \pm 5.17$ & 0.03 \\
\hline & IP-10 & $1.22 \pm 0.08$ & $1.46 \pm 0.06$ & 0.004 \\
\hline & RANTES & $\begin{array}{c}78.92 \\
(76.70,95.84)\end{array}$ & $\begin{array}{c}112.22 \\
(89.72,153.53)\end{array}$ & 0.04 \\
\hline
\end{tabular}

Data expressed as mean \pm SEM or median (IQR), depending on normality of data distribution. Only statistically significant differences are shown. See Methods section for full panel of 24 cytokines and chemokines tested.

$C D$, control diet; HFD, high-fat hypercaloric diet; LL, interleukin; IP, interferon- $\gamma$-induced protein; IQR, interquartile range; MCP, monocyte chemoattractant protein; TGF, transforming growth factor; TNF, tumor necrosis factor. in response to inhaled methacholine at a cumulative dose of $3.5 \mathrm{mg} / \mathrm{ml}(P<0.05)$.

\section{Response to RSV}

After $5 \mathrm{~d}$ of infection at $2 \mathrm{wk}$ of age, RSV titers measured in lung tissues of weanlings born to HFD dams were on average threefold higher than in age-matched controls born to CD dams (Figure 3a; $n=7$ per group). Active viral replication was confirmed by the parallel increase of NGF expression measured in the lungs of the same infected HFD weanlings compared to infected CD controls (Figure $3 \mathbf{b}$ ). Also, the increase of $\mathrm{R}_{\mathrm{rs}}$ in response to aerosolized methacholine challenge was significantly larger in infected HFD weanlings than in infected $\mathrm{CD}$ controls (Figure 3c; $P<0.05$ ).

\section{DISCUSSION}

This study shows that a dietary change in a normal-weight mother during gestation leads to offspring with abnormal metabolic profiles, chronic airway inflammation, and increased airway reactivity predisposing to airflow obstruction and increased susceptibility to RSV infection. Pups born from mothers fed a hypercaloric diet with high fat content during pregnancy were characterized by hypertriglyceridemia, as well as adiposity evidenced by increased body fat without a corresponding change in body weight. Despite being more difficult to measure than the widely used body mass index (BMI), body fat is considered the gold standard to be used in epidemiological studies exploring the association between adiposity and asthma in children (8). Importantly, the postnatal metabolic abnormalities in offspring exposed to HFD during gestation were long-lasting and persisted throughout development.

Paradoxically, offspring born to HFD dams were not large for gestational age, and actually became significantly lighter than controls after the newborn period. This phenomenon can be explained by the many human and animal studies demonstrating a profound impact of maternal diet during pregnancy

Table 3. Significant increase of cytokines and chemokines in serum of offspring

\begin{tabular}{|c|c|c|c|c|}
\hline & Analyte & Maternal control diet (pg/ml) & Maternal high-fat diet (pg/ml) & $P$ value \\
\hline \multirow[t]{2}{*}{ Weanlings; $C D n=7 ; \mathrm{HFD} n=7$} & IL-5 & $5.6 \pm 3.6$ & $288.7 \pm 21.4$ & $<0.001$ \\
\hline & MCP-1 & $793.5(619.2,1039.4)$ & $4311.3(3474.1,5298.0)$ & $<0.001$ \\
\hline Adults; $\mathrm{CD} n=7 ; \mathrm{HFD} n=8$ & Leptin & $44,284.8 \pm 6,151.3$ & $87,238 \pm 9,970.8$ & 0.004 \\
\hline
\end{tabular}

Data expressed as mean \pm SEM or median (IQR), depending on normality of data distribution. Only statistically significant differences are shown. See Methods section for full panel of 24 cytokines and chemokines tested.

CD, control diet; HFD, high-fat hypercaloric diet; IL, interleukin; MCP, monocyte chemoattractant protein; VEGF, vascular endothelial growth factor.

Table 4. Significant increase of cytokines and chemokines in bronchoalveolar lavage of offspring

\begin{tabular}{|c|c|c|c|c|}
\hline & Analyte & Control diet (pg/ml) & High-fat diet (pg/ml) & $P$ value \\
\hline Weanling; CD $n=6 ; \mathrm{HFD} n=6$ & MCP-1 & $370.06 \pm 47.74$ & $777.34 \pm 92.13$ & 0.003 \\
\hline Adult; $\mathrm{CD} n=6 ; \mathrm{HFD} n=6$ & IL-17 & $19.31 \pm 2.18$ & $27.12 \pm 2.43$ & 0.04 \\
\hline
\end{tabular}

Data expressed as mean \pm SEM or median (IQR), depending on normality of data distribution. Only statistically significant differences are shown. See Methods section for full panel of 24 cytokines and chemokines tested.

CD, control diet; HFD, high-fat hypercaloric diet; IL, interleukin; IQR, interquartile range; MCP, monocyte chemoattractant protein; 
a

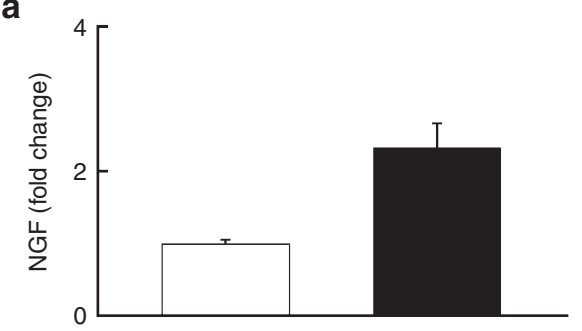

b

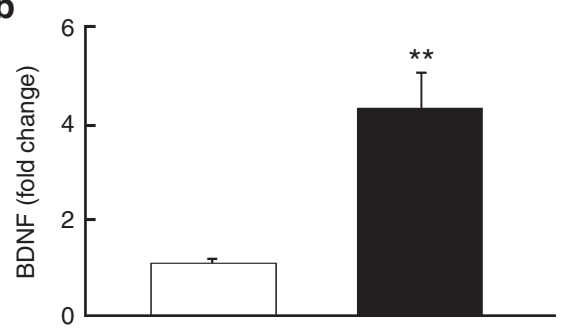

C

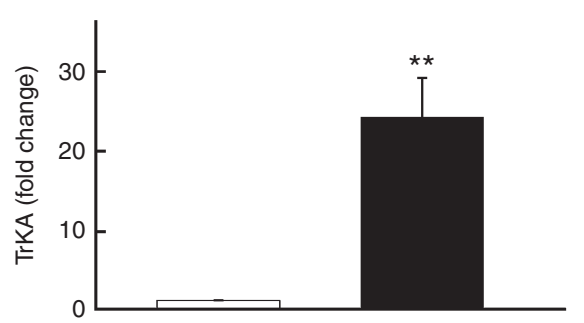

d

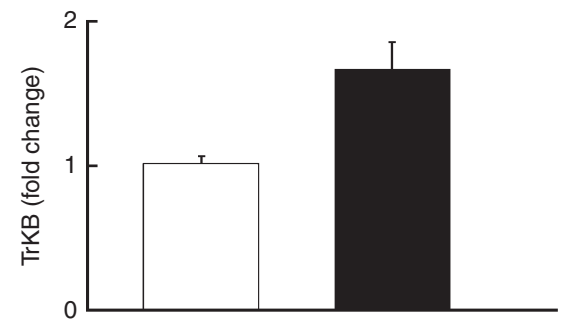

e

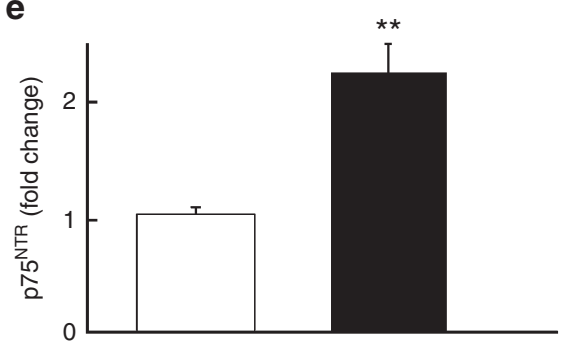

Figure 1. Effect of maternal diet on neurotrophins expression in weanling lungs. Gene expression for the neurotrophic factors (a) NGF and (b) BDNF as well as their respective cognate receptors (c) TrKA, (d) TrKB, and (e) p75 NTR was upregulated in the lung tissues of pups born to dams fed high-fat hypercaloric diet during pregnancy (black fill) compared with age-matched controls born to dams fed a normal diet (no fill). Also increased was the nonspecific pan-neurotrophic receptor $p 75^{\text {NTR }}$. Data are expressed as mean \pm SEM ( $n=7$ rats per group). ${ }^{* *} P<0.01$ compared with age-matched controls born to dams fed with control diet.

a

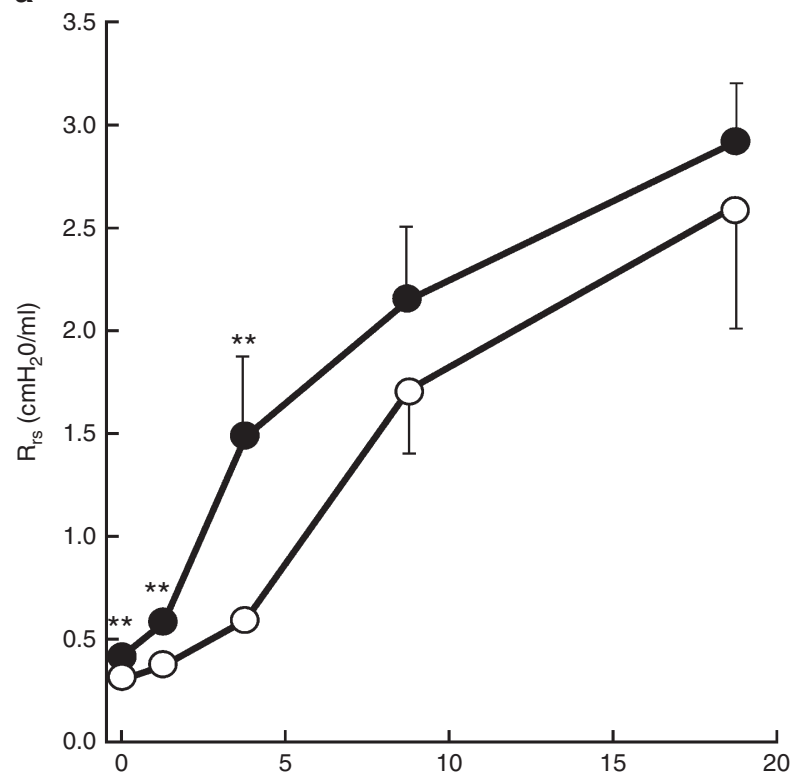

b

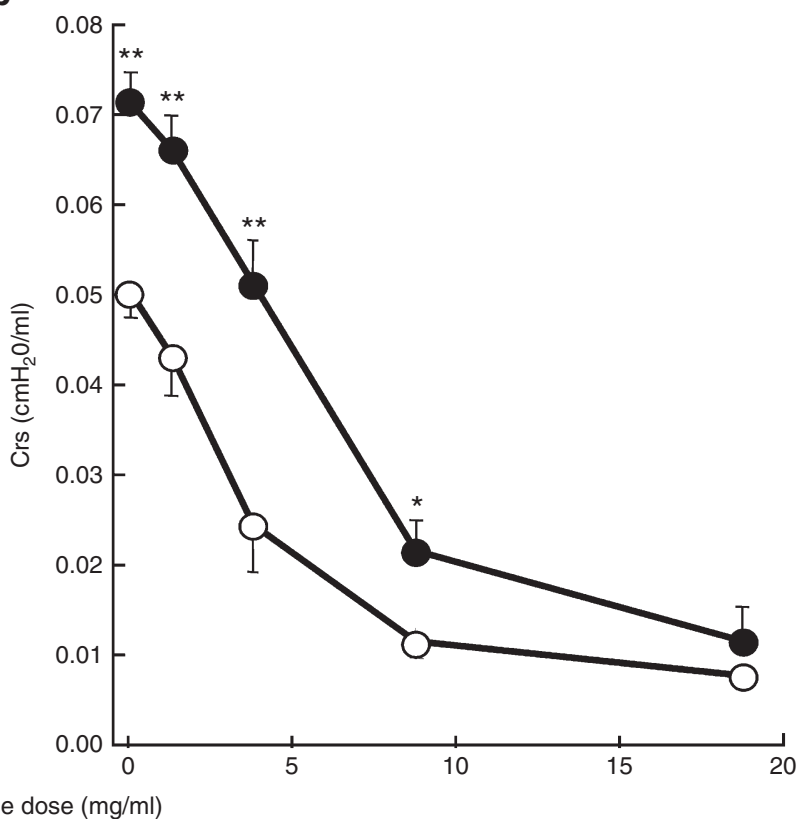

Figure 2. Effect of maternal diet on pulmonary function of weanlings. Weanlings born to dams fed high-fat hypercaloric diet (HFD) during pregnancy (solid circles) had significantly higher $(\mathbf{a})$ respiratory system resistance $\left(R_{r s}\right)$ and lower $(\mathbf{b})$ respiratory system compliance $\left(C_{r s}\right)$ at baseline compared with age-matched controls born to dams fed a normal diet (open circles). Following challenge with aerosolized methacholine, significantly higher airway resistance and lower lung compliance were observed in HFD offspring. Data are expressed as mean \pm SEM $\left(n=7\right.$ rats per group). ${ }^{*} P<0.05 ;{ }^{* *} P<0.01$ compared with age-matched controls born to dams fed with control diet.

on placental function, fetal growth, and postnatal development (9). In the sheep model, overfeeding pregnant adolescent ewes with high quantity of a complete diet resulted in placental and fetal growth restriction during gestation, complex hormonal changes in the mother, and low offspring's birth weight (10). Similarly, an analysis of human fetal outcomes in relation to maternal diet in the Southampton Women's Survey cohort revealed that energy intake in early pregnancy can be inversely related to placental size and birth weight (11).

Together with the weight data, the significantly decreased nutrient concentrations measured in newborns (cholesterol, glucose, and triglycerides) suggest that high-fat hypercaloric 


\section{Articles | Griffiths et al.}
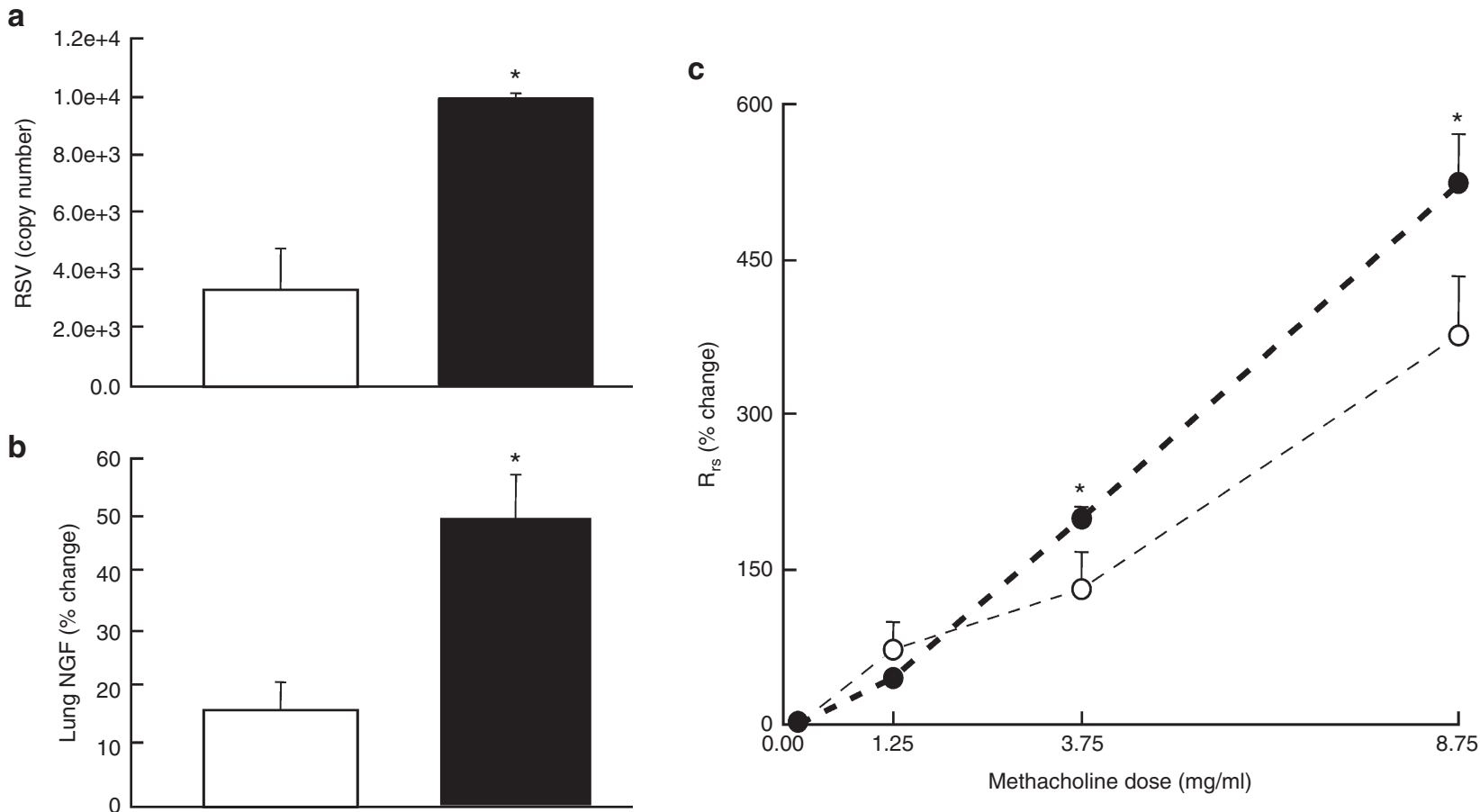

Figure 3. Effect of maternal diet on susceptibility to RSV of weanlings. After $5 \mathrm{~d}$ of infection at 2 wk of age, (a) RSV copy number and (b) change in NGF expression in lung tissues of weanlings born from dams fed high-fat hypercaloric diet (HFD) during pregnancy (black fill) was on average threefold higher than that in age-matched weanlings born from control dams (no fill). Also, (c) increase of respiratory system resistance $\left(R_{r s}\right)$ in response to aerosolized methacholine challenge was significantly larger in infected HFD weanlings (solid circles) than in infected controls (open circles). Data are expressed as mean \pm SEM ( $n=7$ rats per group). ${ }^{*} P<0.05$ compared with age-matched controls born to dams fed with control diet. NGF, nerve growth factor; RSV, respiratory syncytial virus.

nutrition in pregnancy induced placental dysfunction and restricted intrauterine fetal growth, thereby preventing higher birth weight despite the maternal nutritional intake. However, this prenatal nutritional imbalance led to postnatal fat accumulation and hypertriglyceridemia. Differences between our rat model and other experimental models may derive from species-specific differences in placental structure and function; the different study diet, high in fat and calories but low in carbohydrates; or by the age of our dams that were already fully developed at the onset of pregnancy.

In our study, adult offspring were maintained on the same diet as their mother, as we made the assumption that this is what usually happens in real life. However, in a subset of experiments conducted in parallel with the ones described in this article, pups were switched at birth to surrogate mothers fed regular diet without observing any significant change in experimental outcomes (data not shown), which emphasizes the critical importance of a balanced maternal diet for physiologic fetal development. Our data complete previous studies describing a strong association between prepregnancy maternal obesity and the development of childhood asthma (12-14), adding the important notion that even nonobese mothers overeating in pregnancy may experience metabolic changes sufficient to interfere with normal fetal development and leading to chronic and perhaps irreversible health concerns in the offspring.

Perhaps the most important finding of this study is that increased availability of nutrients to the placenta was associated with airway inflammation and hyperreactivity during development. This animal model enables the analysis of lung mechanics in the setting of metabolic abnormalities without obesity as a mechanical confounder and confirms the recently described association of abnormal pulmonary function with elevated triglycerides (3). Furthermore, the Fischer F-344 rat strain has no known genetic predisposition to obesity; therefore, we assume that all metabolic, inflammatory, and pathophysiologic effects described in this paper are directly induced by the dietary changes operated in pregnancy.

Obviously, this study has some limitations. First, we did not investigate variables related to maternal metabolism, such as gestational weight gain. Second, body fat was not measured in newborns because of size-related limitations; we also did not measure blood insulin levels and some other assays could not be performed on all samples because of the small blood volume drawn from pups. Finally, because rats will lower their consumption of a high-energy diet to match their energy requirements, it takes a relatively long period to induce obesity using a moderately high-fat diet. Therefore, in this study, we had to use a diet that would be considered extreme for humans because of the limited time the dams were on such diet prior to delivery $(\sim 3 \mathrm{wk})$.

\section{Inflammation}

Previous data in nonhuman primates have shown that HFD during pregnancy reduces uteroplacental perfusion, induces 
placental inflammation, and alters the normal development of multiple organ systems in the fetus independent of maternal obesity $(15,16)$. In our model, fetal exposure to gestational overnutrition led to increased expression of proinflammatory cytokines in lung tissues. In newborn HFD offspring, we found significant elevations in IL- $1 \alpha$, IL-1 $\beta$, IL-2, TNF- $\alpha$, MCP-1, IL-5, IL-9, and eotaxin. These findings imply a predisposition to abnormal lung parenchymal and airway development, as a number of animal and human studies have demonstrated abnormalities in the offspring respiratory tract after exposure to antenatal inflammatory stimuli, even in the absence of significant postnatal injury.

After the neonatal stage, high IL-5 and MCP-1 concentrations persisted both systemically and locally in the lungs during development of pups exposed to maternal HFD during gestation. The cytokine IL-5 is widely recognized as a regulator of eosinophil proliferation, differentiation, and recruitment and has been positively correlated with severity of asthma $(17,18)$. Unlike the T helper cell type-2 (Th2) polarization characteristic of allergic asthma, in this model we did not find significant elevation of IL-13 in the HFD offspring. Nonetheless, constitutive pulmonary expression of IL-5 in the absence of allergic challenge results in mucous metaplasia, expansion of bronchus-associated lymphoid tissue, enhanced collagen deposition, and extensive airway eosinophilia (19).

The chemokine MCP-1 (C-C motif ligand 2, CCL2) is a critical molecular link between adipose tissue and skeletal muscle, in that it impairs insulin-stimulated glucose uptake in myocytes leading to the development of insulin resistance in obesity. MCP-1 is systemically increased in obese children and adults as well as in patients with type 2 diabetes, and it is implicated in the pathogenesis of insulin resistance, obesity-associated metabolic syndrome, diabetes-related cardiovascular diseases, and diabetic nephropathy. In the lungs, MCP-1 is believed to play a role in the pathogenesis of allergic asthma by recruitment of macrophages and monocytes (20). MCP-1 also contributes to the accumulation of macrophages and monocytes in fat tissue, leading to impaired lipid metabolism and subsequent decrease in lipid uptake from the circulation (21). The resulting elevation of serum triglycerides concentration may result in potentiation of systemic inflammation.

\section{Neurotrophins}

Gene expression of NGF, BDNF, and their respective cognate receptors was significantly upregulated in the lungs of pups born to mothers fed HFD during pregnancy. Neurotrophins play an important role in the pathogenesis of airway hyperreactivity and inflammation because they are essential for development, maintenance, and function of the sensory and motor innervation throughout the respiratory tract (22). This neural network is responsible for regulation of smooth muscle tone, mucous secretion, microvascular flow, and permeability, and also participates in the recruitment of inflammatory cells. As in other animal models described in our previous work $(23,24)$, the upregulation of neurotrophic pathways in pups born to overfed dams led to significantly larger increase in respiratory system resistance and decrease in dynamic compliance in response to methacholine inhalation compared with age-matched controls born to dams fed with a balanced diet.

\section{RSV infection}

Diet plays a crucial role in maintaining optimal immune function. Imbalanced nutrition during gestation-both malnutrition and overnutrition-can affect immune system programming during offspring development with profound and long-term consequences persisting into adulthood, and even intergenerational effects that can be observed in second and third generations fed on a normal diet (7). Therefore, we hypothesized that maternal diet may affect offspring susceptibility to RSV, which is the most common viral illness in infancy and is associated with persistent airway inflammation and hyperreactivity mediated at least in part by increased signaling through the NGF-TrKA axis (25).

Inoculation of RSV in weanlings born to HFD mothers resulted in more efficient viral replication in lung tissues compared with age-matched pups born to dams fed with a balanced diet and then inoculated with the same virus suspension. Higher RSV titers were correlated to greater upregulation of NGF expression-which requires active viral replication (26) - as well as to a stronger bronchospastic response to inhaled methacholine. The increased susceptibility to viral infection is consistent with the Th2 polarization and consequent low expression of interferon- $\gamma$ and other Th1 cytokines discussed above but may also reflect defects in other immune pathways linked to the metabolic derangement induced by imbalanced nutrition during critical developmental windows (7).

\section{Conclusions}

In this study, we developed a new model of airway inflammation and hyperreactivity induced by prenatal dietary imbalance, which cannot be ethically achieved in clinical research. Maternal high-fat hypercaloric diet during pregnancy not only caused persistent metabolic abnormality in the offspring reproducing the same pattern previously shown to be associated to childhood asthma-but it also led to chronic airway inflammation and hyperreactivity measurable throughout development. Furthermore, overfed dams delivered pups prone to develop more severe RSV disease, which is another independent risk factor for recurrent wheezing in childhood (27). Therefore, our findings support the principles outlined in Barker's "Foetal Programming Hypothesis" while suggesting that maternal nutrition during pregnancy is a critical determinant of the risk for bronchiolitis and asthma in childhood, independently from prepregnancy nutrition (28). As these lifestyle characteristics are amenable to change, a greater focus on the diet and metabolic health of pregnant women could have a much greater impact on the global epidemics of childhood obesity and asthma than any much more expensive strategy based on postnatal prevention or therapy. 


\section{Articles | Griffithset al.}

\section{METHODS}

\section{Animals}

All experiments outlined in this study were performed on pathogenfree rats strain Fischer 344 (F-344) and were approved by the West Virginia University Animal Care and Use Committee. Rats were mated overnight using two females per male, and the day on which spermatozoa were found in the vaginal smear was recorded as the first day of gestation (23). On the same day, dams were switched from standard chow to a HFD (Research Diets, New Brunswick, NJ; Table 5). As control diet (CD), we used a matched and purified ingredient diet (American Institute of Nutrition 93G; Research Diets). Terminal experiments were performed on newborn (1-d old), weanling (2-wks old), or adult (12-wks old) offspring of dams fed the study diet during gestation and lactation (Figure 4). Adult offspring were maintained on the same diet as their respective mother.

Following pentobarbital anesthesia $(50 \mathrm{mg} / \mathrm{kg}$ i.p.), the chest was opened and blood collected by cardiac puncture. An aliquot of whole blood was analyzed for glucose and lipid profile (total cholesterol, high-density lipoprotein cholesterol, and triglycerides) with Cholestech LDX (Alere, San Diego, CA). The remaining blood was centrifuged to separate the cellular fraction and frozen at $-80{ }^{\circ} \mathrm{C}$. Lung tissues were dissected, snap-frozen in liquid nitrogen, and stored at $-80^{\circ} \mathrm{C}$ until analysis. Proportional weight of the gonadal fat pads was used as a reliable estimate of body fat in normal and study weanling and adult rats (29), but we were unable to obtain reliable measurements at birth because there was essentially no fat in newborns of either group.

\section{Bronchoalveolar lavage}

Weanling and adult rats were anesthetized with pentobarbital, and a plastic catheter was inserted into the trachea and secured with proximal and distal sutures (30). BAL was performed by instilling and withdrawing $28 \mathrm{ml} / \mathrm{kg}$ of saline into the catheter. This procedure was repeated three times, and the fluid collected from each rat was pooled into a single sample for analysis. BAL could not be performed in newborn pups due to size-related technical limitations.

\section{Cytokines expression}

This analysis was performed on serum, BAL fluid, and lung tissue homogenates. Luminex xMAP technology (Luminex, Austin, TX) was used for multiplex quantification of the following 24 cytokines and chemokines that were simultaneously measured using a Milliplex MAP rat cytokine/chemokine panel (Millipore, Billerica, MA) according to manufacturer specifications: eotaxin, granulocyte colony-stimulating factor, granulocyte macrophage colonystimulating factor, growth-related oncogene/keratinocyte derived chemokine, interferon- $\gamma$, IL-10, IL-12 (p70), IL-13, IL-17, IL-18,

Table 5. Maternal diet composition

\begin{tabular}{|c|c|c|c|c|c|c|c|}
\hline \multirow[b]{2}{*}{ Diet } & \multicolumn{2}{|c|}{ Protein } & \multicolumn{2}{|c|}{ Carbohydrates } & \multicolumn{2}{|c|}{ Fat } & \multirow{2}{*}{$\begin{array}{l}\text { Energy } \\
\mathrm{Kcal} / \mathrm{g}\end{array}$} \\
\hline & Weight\% & Kcal\% & Weight\% & Kcal\% & Weight\% & Kcal\% & \\
\hline Control (AIN-93G) & 20.0 & 20.3 & 64.0 & 63.9 & 7.0 & 15.8 & 3.90 \\
\hline High-fat (60 Kcal \%) & 26.2 & 20.0 & 26.3 & 20.0 & 34.9 & 60.0 & 5.24 \\
\hline
\end{tabular}

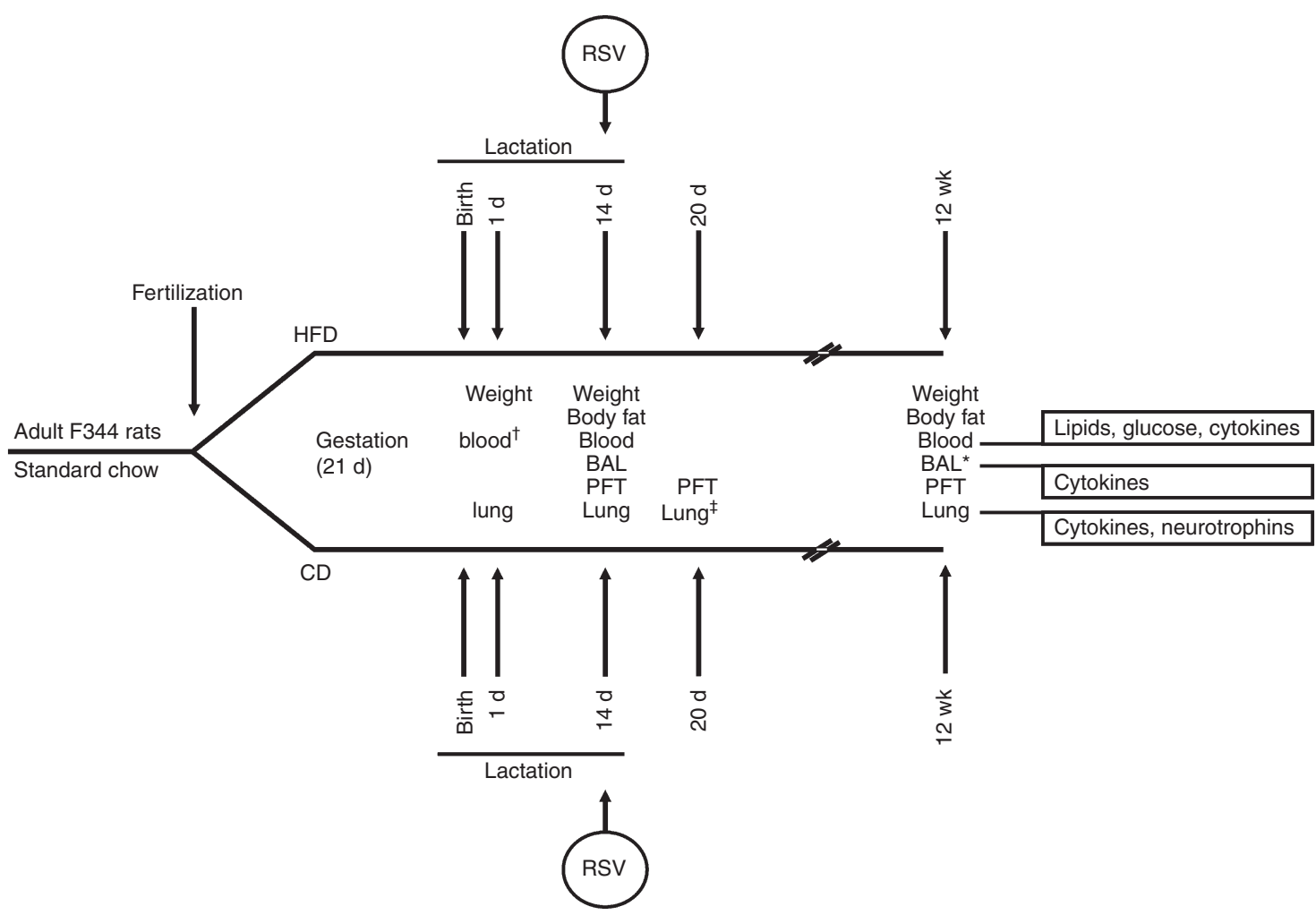

Figure 4. Study design. Pregnant dams were switched on gestation day 1 from standard chow to either high-fat hypercaloric diet (HFD) or control diet (CD). Terminal experiments were performed on newborn (1-d old) and weanling (2-wk old) offspring of dams fed the study diet during gestation and lactation, or adult (12-wk old) offspring maintained on the same diet as their mother. A subgroup of weanlings was infected with respiratory syncytial virus (RSV). BAL, bronchoalveolar lavage; PFT, pulmonary function test. 'Blood collected from newborn rats was analyzed for glucose and lipid profile only; the serum cytokine panel could not be performed due to limited sample volume. "Lungs dissected from weanlings infected with RSV were used only for measurements of RSV titer and NGF expression. NGF, nerve growth factor. 
IL- $1 \alpha$, IL- $1 \beta$, IL-2, IL-4, IL-5, IL-6, IL-9, interferon- $\gamma$-induced protein-10, leptin, MCP-1, macrophage inflammatory protein$1 \alpha$, regulated on activation normal $\mathrm{T}$ cell expressed and secreted, tumor necrosis factor- $\alpha$, and vascular endothelial growth factor. Results were normalized to protein content of tissue homogenate, as determined by Precision Red Assay (Cytoskeleton, Denver, CO). A serum panel was not performed for newborn rats due to the limited sample volume.

\section{Neurotrophins expression}

Lung tissue expression of genes encoding for key neurotrophic factors and their cognate receptors was conducted as described previously (26). Briefly, frozen lung specimens were homogenized in RLT buffer (Qiagen, Valencia, CA). Total RNA was isolated using an RNeasy kit following manufacturer's recommendations, and $100 \mathrm{ng}$ was used as the template for quantitative real-time PCR analysis using Quantitect SYBR green RT-PCR master mix (Qiagen). Samples were run with the ABI 7500 real-time cycler (Applied Biosystems, Foster City, CA). Primers for NGF and BDNF; TrKA and TrKB; and the nonspecific pan-neurotrophin receptor $\mathrm{p} 75^{\mathrm{NTR}}$ were purchased from SuperArray (Rockville, MD), and hypoxanthine phosphoribosyltransferase-1 (Real Time Primers, Elkins Park, PA) was used as the housekeeping gene for transcript normalization. Relative changes in gene expression were calculated with the $2^{-\Delta \Delta C}$ method.

\section{Pulmonary function tests}

The trachea of weanling and adult rats under deep anesthesia was exposed through a neck incision, a small cut was made below the cricoid cartilage, and an 18-gauge catheter was inserted and secured with sutures (23). The catheter was connected via a nebulizer to a Flexivent system (SCIREQ, Montreal, Canada). As in previous studies (23), indirect measurements of $R_{r s}$ and $C_{r s}$ were made at baseline after nebulization of phosphate-buffered saline and then after nebulization of methacholine at cumulative doses of $1.25,3.75,8.75$, and $18.75 \mathrm{mg} / \mathrm{ml}$, or until a $200 \%$ increase in $\mathrm{R}_{\mathrm{rc}}$ was noted. This protocol could not be performed in newborn pups due to size-related technical limitations.

\section{RSV infection}

Weanling rats were lightly anesthetized with methohexital sodium and $40 \mu \mathrm{l}$ of red fluorescent protein-tagged RSV $\left(1.8 \times 10^{5} \mathrm{TCID}_{50}\right)$ was inoculated by intratracheal instillation as described previously (23). Pulmonary function tests were performed as described above at $5 \mathrm{~d}$ post-inoculation, and the lungs were dissected immediately after for measurements of RSV titer and NGF expression. RSV copy number in the lungs was measured using a One-Step RSV-A Quantitation Kit (Primer Design, Southampton, UK) as per manufacturer's instructions.

\section{Statistical Analysis}

Data are expressed as mean \pm SEM or median (IQR), depending on normality of distribution. Differences among groups were analyzed by one-way ANOVA. Statistical analysis was performed using the software SigmaStat version 3.5 (Systat, Point Richmond, CA). Differences with $P<0.05$ were considered significant.

\section{ACKNOWLEDGMENTS}

We are indebted to Mark Peeples (Nationwide Children's Hospital Research Institute, Columbus, $\mathrm{OH}$ ) and Peter Collins (National Institutes of Health, Bethesda, MD) for providing the original batch of the red fluorescent protein-expressing RSV virus. We also thank Janet Tou (West Virginia University Davis College of Agriculture, Natural Resources and Design) for providing the study and control diets and for consulting on other nutritional aspects of this project.

\section{STATEMENT OF FINANCIAL SUPPORT}

This study was funded in part by a grant from the US National Institutes of Health, National Heart, Lung, and Blood Institute (Bethesda, MD) grant RO1 HL-61007 to G.P.

Disclosure: The authors declare no conflict of interest.

\section{REFERENCES}

1. Ogden CL, Carroll MD, Kit BK, Flegal KM. Prevalence of childhood and adult obesity in the United States, 2011-2012. JAMA 2014;311: 806-14.

2. Shore SA. Obesity and asthma: possible mechanisms. J Allergy Clin Immunol 2008;121:1087-93; quiz 1094-5.

3. Cottrell L, Neal WA, Ice C, Perez MK, Piedimonte G. Metabolic abnormalities in children with asthma. Am J Respir Crit Care Med 2011;183: 441-8.

4. Greenough A. Prenatal factors in the development of chronic lung disease. Semin Fetal Neonatal Med 2009;14:339-44.

5. Holguin F, Fitzpatrick A. Obesity, asthma, and oxidative stress. J Appl Physiol (1985) 2010;108:754-9.

6. de Vries A, Hazlewood L, Fitch PM, Seckl JR, Foster P, Howie SE. High-fat feeding redirects cytokine responses and decreases allergic airway eosinophilia. Clin Exp Allergy 2009;39:731-9.

7. Pérez-Cano FJ, Franch À, Castellote C, Castell M. The suckling rat as a model for immunonutrition studies in early life. Clin Dev Immunol 2012;2012:537310.

8. Yiallouros PK, Lamnisos D, Kolokotroni O, Moustaki M, Middleton N. Associations of body fat percent and body mass index with childhood asthma by age and gender. Obesity (Silver Spring) 2013;21: E474-82.

9. Thornburg KL, O’Tierney PF, Louey S. Review: The placenta is a programming agent for cardiovascular disease. Placenta 2010;31:Suppl:S54-9.

10. Wallace JM, Aitken RP, Milne JS, Hay WW Jr. Nutritionally mediated placental growth restriction in the growing adolescent: consequences for the fetus. Biol Reprod 2004;71:1055-62.

11. Godfrey K, Robinson S, Barker DJ, Osmond C, Cox V. Maternal nutrition in early and late pregnancy in relation to placental and fetal growth. BMJ 1996;312:410-4.

12. Håberg SE, Stigum H, London SJ, Nystad W, Nafstad P. Maternal obesity in pregnancy and respiratory health in early childhood. Paediatr Perinat Epidemiol 2009;23:352-62.

13. Patel SP, Rodriguez A, Little MP, et al. Associations between pre-pregnancy obesity and asthma symptoms in adolescents. J Epidemiol Community Health 2012;66:809-14.

14. Scholtens S, Wijga AH, Brunekreef B, et al. Maternal overweight before pregnancy and asthma in offspring followed for 8 years. Int J Obes (Lond) 2010;34:606-13.

15. Frias AE, Morgan TK, Evans AE, et al. Maternal high-fat diet disturbs uteroplacental hemodynamics and increases the frequency of stillbirth in a nonhuman primate model of excess nutrition. Endocrinology 2011;152:2456-64.

16. McCurdy CE, Bishop JM, Williams SM, et al. Maternal high-fat diet triggers lipotoxicity in the fetal livers of nonhuman primates. J Clin Invest 2009;119:323-35.

17. Wu CA, Peluso JJ, Zhu L, Lingenheld EG, Walker ST, Puddington L. Bronchial epithelial cells produce IL-5: implications for local immune responses in the airways. Cell Immunol 2010;264:32-41.

18. Kay AB, Phipps S, Robinson DS. A role for eosinophils in airway remodelling in asthma. Trends Immunol 2004;25:477-82.

19. Lee JJ, McGarry MP, Farmer SC, et al. Interleukin-5 expression in the lung epithelium of transgenic mice leads to pulmonary changes pathognomonic of asthma. J Exp Med 1997;185:2143-56.

20. Rose CE Jr, Sung SS, Fu SM. Significant involvement of CCL2 (MCP-1) in inflammatory disorders of the lung. Microcirculation 2003; 10:273-88

21. Norata GD, Grigore L, Raselli S, et al. Triglyceride-rich lipoproteins from hypertriglyceridemic subjects induce a pro-inflammatory response in the endothelium: Molecular mechanisms and gene expression studies. J Mol Cell Cardiol 2006;40:484-94.

22. Scuri M, Samsell L, Piedimonte G. The role of neurotrophins in inflammation and allergy. Inflamm Allergy Drug Targets 2010;9:173-80.

23. Piedimonte G, Walton C, Samsell L. Vertical transmission of respiratory syncytial virus modulates pre- and postnatal innervation and reactivity of rat airways. PLoS One 2013;8:e61309. 


\section{Articles | Grifithsetal.}

24. Scuri M, Chen BT, Castranova V, et al. Effects of titanium dioxide nanoparticle exposure on neuroimmune responses in rat airways. J Toxicol Environ Health A 2010;73:1353-69.

25. Piedimonte G. Respiratory syncytial virus and asthma: speed-dating or long-term relationship? Curr Opin Pediatr 2013;25:344-9.

26. Hu C, Wedde-Beer K, Auais A, Rodriguez MM, Piedimonte G. Nerve growth factor and nerve growth factor receptors in respiratory syncytial virus-infected lungs. Am J Physiol Lung Cell Mol Physiol 2002;283: L494-502.

27. Piedimonte G, Perez MK. Respiratory syncytial virus infection and bronchiolitis. Pediatr Rev 2014;35:519-30.

28. Barker DJ. The origins of the developmental origins theory. J Intern Med 2007;261:412-7.

29. Rogers P, Webb GP. Estimation of body fat in normal and obese mice. Br J Nutr 1980;43:83-6.
30. Auais A, Adkins B, Napchan G, Piedimonte G. Immunomodulatory effects of sensory nerves during respiratory syncytial virus infection in rats. Am J Physiol Lung Cell Mol Physiol 2003;285:L105-13.

This work is licensed under a Creative Commons Attribution-NonCommercial-NoDerivs International License. The images or other third party material in this article are included in the article's Creative Commons license, unless indicated otherwise in the credit line; if the material is not included under the Creative Commons license, users will need to obtain permission from the license holder to reproduce the material. To view a copy of this license, visit http://creativecommons.org/licenses/by-nc-nd/4.0/ 